\title{
A INSERÇÃO INTERNACIONAL DAS EMPRESAS FARMACÊUTICAS NACIONAIS
}

\author{
Fernanda Steiner Perin ${ }^{1}$ \\ Julia Paranhos ${ }^{2}$
}

\begin{abstract}
Área 2 - Comércio internacional, cadeias de valor e internacionalização
2.1 - Investimento Direto Estrangeiro e internacionalização de empresas
\end{abstract}

\begin{abstract}
RESUMO
O objetivo deste trabalho é identificar os principais movimentos de inserção internacional das empresas farmacêuticas brasileiras de capital nacional. A metodologia para o tema proposto utiliza fontes de dados secundárias por meio de pesquisas bibliográficas e documentais. Por meio dos dados de comércio exterior, encontrou-se que a balança comercial da indústria farmacêutica é estruturalmente deficitária. As importações tiveram altas taxas de crescimento devido à ampliação da oferta dos insumos farmacologicamente ativos (IFAs), oriundas dos países asiáticos, e dos medicamentos prontos, sobretudo, os de base biotecnológica, pelo extenso período de apreciação da moeda brasileira e pelo crescimento do mercado consumidor doméstico. Por sua vez, foi evidenciado um crescimento das exportações da indústria farmacêutica a partir do início dos anos 2000, juntamente com o crescimento das empresas nacionais a partir da Lei dos Medicamentos Genéricos. Contudo, embora as exportações da indústria farmacêutica brasileira tenham apresentado altas taxas de crescimento, os produtos brasileiros ainda são de baixo valor agregado. Foi observado um movimento de inserção externa das empresas farmacêuticas nacionais que empreendem estratégias de internacionalização em duas direções. Por um lado, as empresas farmacêuticas de capital nacional adotam a estratégia de acessar os mercados dos países latino-americanos e, por outro lado, estão se inserindo em países desenvolvidos para buscar capacitação tecnológica.
\end{abstract}

Palavras-Chave: empresa farmacêutica brasileira; estratégia de internacionalização; capacitação tecnológica.

\begin{abstract}
The work aims to identify the main movements of international insertion of Brazilian national-owned firms of the pharmaceutical industry. The methodology for the proposed theme uses sources of secondary data through bibliographical and documentary research. Through the data of foreign trade, it was found that the balance of trade of the pharmaceutical industry has a structurally deficient. Imports had high growth rates due to the expansion of the supply of active pharmaceutical ingredients (API) from Asian countries, and of finished drugs, mainly biotechnological ones, due to the long period of appreciation of the Brazilian currency and the growth of the market consumer. In turn, there was an increase in the exports of the Brazilian pharmaceutical industry from the beginning of the 2000s, together with the growth of nationalowned firms since the Law of Generic Drugs. However, although exports from the Brazilian pharmaceutical industry showed high growth rates, Brazilian products still have low value added. It was observed a foreign insertion of the pharmaceutical Brazilian national-owned firms that undertake strategies of internationalization in two directions. On the one hand, nationally-owned pharmaceutical firms adopt the strategy of accessing the markets of Latin American countries and, on the other hand, are entering into developed countries to seek technological capacity.
\end{abstract}

Keywords: Brazilian pharmaceutical firm; internationalization strategy; technological capacity.

\section{JEL: F23; L13; L65.}

\footnotetext{
${ }^{1}$ Doutoranda em Economia da Indústria e da Tecnologia pelo Instituto de Economia da Universidade Federal do Rio de Janeiro (IE/UFRJ). E-mail: fernanda.steinerperin@ gmail.com.

${ }^{2}$ Professora Adjunta do Instituto de Economia da Universidade Federal do Rio de Janeiro (IE/UFRJ). E-mail: juliaparanhos@ie.ufrj.br.
} 


\section{INTRODUÇÃO}

A indústria farmacêutica é uma atividade com altas taxas de crescimento, globalizada, intensiva em tecnologia e baseada em ciência, conforme a taxonomia de Pavitt (1984). A internacionalização desta indústria é um processo existente desde sua formação e parte da sua dispersão geográfica é resultado dos choques tecnológicos e institucionais que impactaram na sua estrutura produtiva. A emergência da biotecnologia, exigências mais rigorosas na aprovação de novos medicamentos e questões envolvendo direitos de propriedade intelectual são fatores que têm contribuído para acelerar sua globalização. Estes fatores levaram a indústria farmacêutica a passar, nos últimos anos, por uma reestruturação do seu processo produtivo com a terceirização das atividades de Pesquisa \& Desenvolvimento (P\&D), marketing e comercialização, a qual impacta nas capacidades e estratégias das empresas. A indústria farmacêutica é uma das mais internacionalizadas e que mais movimenta os negócios internacionais. Em 2015, o valor das Fusões \& Aquisições (F\&A) transfronteiriça da indústria farmacêutica somou US\$ 149,5 bilhões, o que representa $21 \%$ do total das F\&A e $41 \%$ das F\&A da indústria de transformação (UNCTAD, 2016).

As empresas farmacêuticas são condicionadas a buscar níveis elevados de produtividade e investimentos em inovação por meio de parcerias a fim de estarem orientadas à dinâmica do mercado global (RADAELLI, 2006). Uma forte concorrência nos mercados domésticos e exposição à concorrência internacional permitem um melhor desempenho inovador e níveis mais elevados de competitividade (MCKELVEY; ORSENIGO, 2001). Por estes motivos, as grandes empresas farmacêuticas atuam em escala mundial, estando presentes em praticamente todos os mercados. Esta situação de competitividade em nível global afeta as estratégias de crescimento das empresas, incitando a busca pela atuação internacional.

$\mathrm{Na}$ literatura sobre a internacionalização da firma estão identificados diversos fatores que conferem vantagens competitivas às empresas que buscam se inserir no exterior. Assim, as vantagens competitivas são definidas como um conjunto de recursos e capacidades que diferenciam uma empresa em relação às suas concorrentes atuantes no mercado hospedeiro. Um desses fatores são as capacidades dinâmicas, que são os processos (rotinas) e recursos (posições) únicos para cada firma, e se referem à capacidade de inovar, adaptar e competir em uma rede de negócios em constante mudança (TEECE, 2007; 2014).

Dunning (1988) explana que as empresas que buscam a internacionalização possuem vantagens específicas da propriedade das firmas e vantagens de localização. As vantagens específicas da propriedade das firmas referem-se às vantagens competitivas que a firma pode criar, como determinados tipos de tecnologia ou habilidades organizacionais, ou ainda, aquelas que a firma pode comprar de outras instituições e, desta forma, ter direitos de propriedade sobre estes recursos. As vantagens de localização são aquelas que impõem barreiras à importação e incentivam os investimentos, como o custo de mão de obra e material e políticas governamentais. A propensão da empresa em explorar as vantagens de propriedade e de localização consiste na vantagem de internalização do capital, tecnologia e habilidades gerenciais para produzir em determinado país ao invés de externalizá-los através de investimentos em portfólio, licenciamento ou outro.

Em termos gerais, pode-se dizer que as empresas internacionalizam suas atividades por dois motivos: para explorar seus ativos e capacidades competitivas existentes (asset-exploiting/home-base exploiting activity) ou para aprimorar seus ativos e capacidades (asset-augmenting/home-base augmenting activity). $\mathrm{O}$ primeiro tipo significa usar os recursos e vantagens tecnológicas existentes no país de origem para aumentar a criação de valor no país estrangeiro, enquanto que o segundo visa melhorar os ativos existentes, adquirir (e internalizar) ou criar novos ativos complementares por meio das atividades realizadas no exterior. Ambos são uma combinação de estratégias das empresas transnacionais (ETNs) com as condições de um mercado estrangeiro específico (DUNNING, 2000; KUEMMERLE, 1999; NARULA; ZANFEI, 2004).

Dunning (2000) definiu quatro tipos amplos de estratégias de empresas na internacionalização: acesso ao mercado, para alcançar um mercado externo específico ou uma demanda orientada a um tipo de mercado; acesso a recursos, como financeiros, naturais e mão de obra especializada; acesso à eficiência, para a especialização em uma carteira de ativos estrangeiros e nacionais; e, acesso a ativos estratégicos, para proteger ou amentar as vantagens de propriedade específicas da empresa e/ou reduzir as de seus concorrentes. 
Le Bas e Patel (2007), Patel e Vega (1999) e Le Bas e Sierra (2002) ainda identificaram três variedades da estratégia de acessar ativos estratégicos, que se baseiam no perfil tecnológico do país de origem e de acolhimento da ETN. Dentre elas, uma segue a motivação de exploração de ativos (explorar a P\&D e tecnologia do país de origem) e duas são motivadas pelo aprimoramento de ativos (busca por tecnologia e aumentar a P\&D e tecnologia do país de origem).

A empresa que realiza Investimento Direto Externo (IDE) para explorar suas vantagens competitivas em uma área tecnológica procura regiões/países que são fracas nesta área (KUEMMERLE, 1999). Patel e Pavitt (1990 apud LE BAS; PATEL, 2007) afirmam que o IDE para explorar as capacidades tecnológicas da empresa-matriz do país de origem está apenas interessado em aumentar a rentabilidade da empresa no curto prazo, sem tentar melhorar seu capital tecnológico através do investimento. De forma oposta, a estratégia direcionada a compensar as fraquezas da empresa-matriz em uma determinada área tecnológica selecionando um país anfitrião com força comprovada na tecnologia desejada refere-se ao IDE em busca de tecnologia (LE BAS; PATEL, 2007). Esta é realizada por meio da criação de uma filial de P\&D no país de acolhimento ou pela aquisição de tecnologia estrangeira (PATEL; VEGA, 1999).

Por sua vez, o IDE realizado para aumentar as capacidades tecnológicas da empresa-matriz é realizado quando ela possui alguma vantagem competitiva no país de origem e se instala em países fortes tecnologicamente. Neste caso, a ETN procura adquirir vantagens competitivas que sejam complementares àquelas que já possui, de modo a aumentar seu estoque de conhecimento existente (LE BAS; PATEL, 2007).

Especialmente para o motivo de aprimoramento de ativos e capacidades, o contexto do local é considerado uma fonte de competências e oportunidades tecnológicas. Quando o conhecimento relevante para a atividade inovativa está aglomerado em determinadas áreas geográficas, as subsidiárias externas buscam o aprimoramento de ativos nestas áreas com o objetivo de se beneficiar dos efeitos de transbordamento de conhecimentos externos gerados pela concentração da produção e das atividades inovativas (NARULA; ZANFEI, 2004, p. 327).

A internacionalização motivada pelo aprimoramento de ativos e capacidades intensificou-se com a proeminência da internacionalização da P\&D e de atividades tecnológicas. Notam-se casos crescentes de subsidiárias que implantam suas atividades tecnológicas estrangeiras em áreas que a empresa possui desvantagem. Isto ocorre porque a empresa entende a internacionalização como uma oportunidade para aprender e desenvolver a ciência e tecnologia desenvolvida no exterior (SERAPIO et al. 2000 apud LE BAS; PATEL, 2007).

Os motivos que guiam a internacionalização são diferentes entre ETNs de países desenvolvidos e em desenvolvimento. As ETNs tradicionais dos países desenvolvidos, em geral, se expandiram no exterior a fim de explorar as vantagens competitivas que desenvolveram em seus mercados domésticos, como a posse de ativos intangíveis (VERNON, 1966; BUCLEY; CASSON, 1976; RUGMAN, 1981), enquanto que a relativa falta de vantagens competitivas de países em desenvolvimento demonstra a importância das vantagens do mercado externo em determinar o padrão de seu IDE.

Ramamurti (2009) descreve que as ETNs dos países em desenvolvimento tiveram menos tempo para aperfeiçoar suas vantagens competitivas e se internacionalizaram para aprimorar tais vantagens e utilizalas no seu país de origem. O ambiente global, marcado pela liberalização dos fluxos financeiros e a revolução das tecnologias de informação e comunicação, proporcionou a aceleração do processo de internacionalização das empresas de países em desenvolvimento, que passaram a explorar as diferenças, ao invés das similaridades, na expansão internacional.

Dunning e Lundan (2008) apontam que devido à necessidade de as ETNs dos países em desenvolvimento criarem capacidades necessárias para absorverem o conhecimento de empresas estrangeiras, frequentemente seus IDEs combinam elementos de exploração de ativos, como a busca de mercado, com o aprimoramento de ativos. Por sua vez, Cuervo-Cazurra e Genc (2008) identificaram que as ETNs latinoamericanas estão seguindo a estratégia de aprimoramento das capacidades reorganizando sua cadeia de 
valor em países avançados para amenizar suas desvantagens comparativas relacionadas ao país de origem, como fracas instituições e tecnologias desatualizadas.

Neste contexto, o objetivo deste trabalho é identificar as principais iniciativas de inserção internacional das empresas farmacêuticas brasileiras de capital nacional. Para tanto, o trabalho está dividido em quatro seções: na primeira constam os aspectos metodológicos e a segunda faz uma contextualização do quadro atual da indústria farmacêutica brasileira. As características da inserção externa da indústria farmacêutica brasileira, bem como, as estratégias das empresas farmacêuticas nacionais estão presentes na seção três. Por fim, na seção quatro encontram-se as conclusões.

\section{METODOLOGIA}

Diante do objetivo deste trabalho - identificar as principais iniciativas de inserção internacional das empresas farmacêuticas brasileiras de capital nacional -, esta pesquisa é classificada como exploratória, pois pretende aprimorar o conhecimento sobre o tema proposto. Utilizam-se os procedimentos técnicos de pesquisa bibliográfica, em livros e artigos sobre as características estruturais e concorrenciais da indústria farmacêutica, bem como, sobre o arcabouço institucional que a delineia, e de pesquisa documental, em relatórios e website das empresas buscando informações sobre a inserção das empresas farmacêuticas no mercado estrangeiro por meio de exportações, acordos colaborativos, licenciamento, F\&A com empresas estrangeiras e IDE.

Nas bases estatísticas da Secretaria de Comércio Exterior (Secex) do Ministério do Desenvolvimento, Indústria e Comércio Exterior (MDIC) foram coletados os dados de exportação e importação dos produtos farmacêuticos. O procedimento de coleta foi realizado por Nomenclatura Brasileira de Mercadoria (NBM) a 10 dígitos, para os anos de 1991 a 1996, e por Nomenclatura Comum do Mercosul (NCM) a 8 dígitos, para os anos de 1997 a 2014, e traduzidos para a Classificação Nacional de Atividade Econômica (CNAE) da versão 2.0, conforme tradutor disponibilizado pela Comissão Nacional de Classificação (Concla) do Instituto Brasileiro de Geografia e Estatística (IBGE). Em seguida, os dados foram classificados em produtos farmoquímicos, referente à CNAE 21.1, e medicamentos, referente à CNAE 21.2. Assim, as fontes de informação utilizadas neste trabalho são secundárias e os tipos de dados são de caráter qualitativo e quantitativos.

Por se tratar de um fenômeno recente, a literatura a respeito da internacionalização de empresas farmacêuticas nacionais é incipiente. Deste modo, a contribuição deste trabalho consiste na sistematização das informações que indicam a direção que este fenômeno está tomando. Devido à carência de evidências para analisar as questões que emergem da problemática levantada, uma pesquisa de campo está sendo planejada com o propósito de investigar detalhadamente as formas de entrada, estratégias, vantagens competitivas e obstáculos encontrados pelas empresas farmacêuticas nacionais no processo de inserção internacional.

\section{A INDÚSTRIA FARMACÊUTICA BRASILEIRA}

O marco institucional para a indústria farmacêutica sofreu diversas alterações a partir da década de 1990. Já no início deste período, a abertura econômica proporcionou a entrada massiva de medicamentos e insumos farmacêuticos devido à redução das barreiras tarifárias e não-tarifárias, dentre as quais, os Programas Especiais de Importação e Lei do Similar Nacional. Esta última atribuía uma taxa na importação de produtos com similar nacional (PINHEIRO; ALMEIDA, 1994). A entrada do Brasil na Organização Mundial do Comércio (OMC) em 1994, conduziu também à adesão ao Acordo TRIPS (Trade-Related Aspects of Intellectual Property Rights Agreement, em Português, Aspectos dos Direitos de Propriedade Intelectual Relacionados ao Comércio). Embora o prazo para os países-membros adequarem suas legislações de direitos de propriedade intelectual aos padrões internacionais fosse de 10 anos, o Brasil promulgou a Lei de Propriedade Intelectual ( ${ }^{\circ}$ 9.279) em 1996. A nova legislação estabeleceu o retorno 
das patentes de medicamentos e processos químicos e demais adequações à padronização internacional e a patenteabilidade de produtos biotecnológicos (URRACA-RUIZ; PARANHOS, 2012).

Ainda buscando regulamentar o setor farmacêutico segundo os padrões internacionais foi aprovada a Política Nacional de Medicamentos, a qual seguia as diretrizes da Organização Mundial da Saúde (OMS) (BERMUDEZ et al., 2000). No âmbito dessa Política, em 1999 foram implementadas a Lei no 9.782, que criou o Sistema Nacional de Vigilância Sanitária e a Agência Nacional de Vigilância Sanitária (Anvisa), e a Lei dos Genéricos (n 9.787), que estabeleceu o segmento de medicamentos genéricos no Brasil (BRASIL, 1999). O ingresso na produção de medicamentos genéricos exigiu das empresas um grande esforço de capacidade tecnológica para atender os requisitos de bioequivalência e biodisponibilidade ${ }^{3}$.

O crescimento das empresas farmacêuticas nacionais foi impulsionado pela especialização na produção de medicamentos genéricos, que abriu um novo segmento possível de atuação para as empresas nacionais. Tais empresas, que tinham ganhado mercado com a comercialização de produtos de ETNs e o desenvolvimento de produtos similares, voltaram-se para a criação de capacitação em engenharia reversa e bioequivalência e biodisponibilidade para produção e comercialização de medicamentos genéricos. $\mathrm{O}$ número de empresas produtoras de genéricos cresceu quase oito vezes e o número de produtos foi ampliado, aproximadamente, 20 vezes entre 2000 e 2008 (IMS HEALTH, 2008 apud FINOTTI, 2008).

Cabe ressaltar que a Lei dos Genéricos significou um obstáculo para muitas empresas, uma vez que os requisitos da nova regulação exigiram capacidades que as empresas nacionais não tinham adquirido até então. Algumas empresas saíram do mercado e outras permaneceram especializadas na fabricação de medicamentos similares, os quais, naquele momento, não precisavam dos mesmos testes que os medicamentos genéricos. ${ }^{4}$

Na retomada de uma política industrial mais ativa a partir de 2000, a saber: Política Industrial, Tecnológica e de Comércio Exterior (PITCE), Política de Desenvolvimento Produtivo (PDP) e Plano Brasil Maior $(\mathrm{PBM})$ - o setor farmacêutico passou a ser considerado estratégico e foco de leis e programas para alavancar seu fortalecimento e capacidade inovativa ${ }^{5}$.

O mercado farmacêutico brasileiro é formado por empresas públicas e privadas de capital nacional e estrangeiro, além de 18 laboratórios farmacêuticos públicos que produzem exclusivamente para o mercado público do Sistema Único de Saúde (SUS). Assim como no mercado internacional, a estrutura da oferta no Brasil é definida como um oligopólio diferenciado, em que a competição ocorre via diferenciação de produto e, não, por preços (HASENCLEVER et al., 2010).

As empresas nacionais ganharam importância na atual configuração do mercado farmacêutico brasileiro, sendo que as duas maiores (EMS e Hypermarcas) são de capital nacional e compreendem mais de $22 \%$ do mercado. Não obstante, a participação das ETNs é proeminente, uma vez que 12 entre as 20 maiores empresas são estrangeiras, conforme ilustra a Tabela 1. Em comparação com o mercado mundial, em que as 20 maiores empresas farmacêuticas compreendem $64 \%$ do faturamento total desta indústria, o mercado brasileiro se mostra bastante concentrado, cuja parcela corresponde a $74 \%$, conforme os dados do IMS Health (2015 apud HYPERMARCAS, 2016a).

\footnotetext{
${ }^{3}$ Biodisponibilidade é definida na lei 9.787 como a velocidade e a extensão de absorção de um princípio ativo em uma forma de dosagem. Na mesma lei, bioequivalência é definida como a equivalência farmacêutica entre produtos apresentados sob a mesma forma farmacêutica, contendo idêntica composição qualitativa e quantitativa de princípios ativos, e que tenham comparável biodisponibilidade.

${ }^{4}$ Posteriormente, a Anvisa estabeleceu o prazo até 2014 para que as fabricantes de medicamentos similares apresentassem os mesmos testes de equivalência exigidos aos medicamentos genéricos, assim acabando com a diferença técnica entre estes dois tipos. Os medicamentos similares passaram a ser indicados como intercambiáveis aos medicamentos de referência e as empresas que não fizessem os testes deveriam registrar os produtos como novos.

${ }^{5}$ Para informações mais detalhadas sobre as políticas e programas para o setor farmacêutico consultar Hasenclever et al. (2016), sobretudo os capítulos 3 e 5 .
} 
Tabela 1 - Principais empresas do mercado farmacêutico* brasileiro, 2015

\begin{tabular}{ccccc}
\hline Ranking & Empresa & Origem do Capital & Faturamento (em R\$ milhões) & Participação de mercado \\
\hline 1 & EMS & Nacional & $9.023,3$ & $12,0 \%$ \\
2 & Hypermarcas & Nacional & $7.662,5$ & $10,2 \%$ \\
3 & Sanofi & Internacional & $6.073,2$ & $8,1 \%$ \\
4 & Novartis & Internacional & $4.492,9$ & $6,0 \%$ \\
5 & Aché & Nacional & $4.311,3$ & $5,7 \%$ \\
6 & Eurofarma & Nacional & $3.835,9$ & $5,1 \%$ \\
7 & Takeda Pharma & Internacional & $2.095,4$ & $2,8 \%$ \\
8 & Bayer & Internacional & $2.002,2$ & $2,7 \%$ \\
10 & Teuto Brasileiro & Nacional & $1.846,3$ & $2,4 \%$ \\
12 & GlaxoSmithKline & Internacional & $1.748,2$ & $2,3 \%$ \\
9 & Pfizer & Internacional & $1.715,9$ & $2,3 \%$ \\
11 & MSD & Internacional & $1.459,7$ & $1,9 \%$ \\
16 & Johnson \& Johnson & Internacional & $1.398,6$ & $1,9 \%$ \\
18 & Merck & Internacional & $1,363,8$ & $1,8 \%$ \\
13 & Biolab & Nacional & $1.360,9$ & $1,8 \%$ \\
15 & Libbs & Nacional & $1.198,5$ & $1,6 \%$ \\
14 & Boehringering & Internacional & $1,6 \%$ \\
17 & Astrazeneca Brasil & Internacional & $1.183,9$ & $1,4 \%$ \\
19 & União Química & Nacional & $1.052,2$ & $1,4 \%$ \\
20 & Abbott & Internacional & $1.030,4$ & $1,1 \%$ \\
& Subtotal 20 maiores & & 822,6 & $\mathbf{7 3 , 8 \%}$ \\
& Outras & & $\mathbf{5 5 . 6 7 7 , 7}$ & $\mathbf{2 6 , 2 \%}$
\end{tabular}

Fonte: IMS Health - PMB em Dez/2015 apud Hypermarcas (2016a).

*Nota: inclui mercado farmacêutico total $=$ medicamentos isentos de prescrição $($ MIPs $)+$ medicamentos controlados + medicamentos genéricos.

Os anos 2000 caracterizam a consolidação, fortalecimento e crescimento do setor farmacêutico brasileiro.

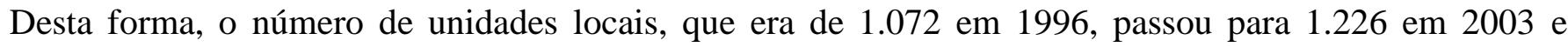
decresceu para 705 em 2014 (IBGE, 2017).

O volume produzido pela indústria farmacêutica também ampliou expressivamente $-41 \%$ entre $1996 \mathrm{e}$ 2016 -, sobretudo, em comparação com o total da indústria de transformação brasileira - 6\% na mesma base de comparação. Esta evolução não é percebida quando analisado o Valor da Transformação Industrial (VTI), variável utilizada como uma proxy do valor adicionado na produção. No período entre 1996 e 2014, o VTI da indústria farmacêutica recuou $8 \%$ e a indústria de transformação cresceu 78\% (IBGE, 2017). Neste ponto, vale considerar a interpretação de Torres e Cavalieri (2015) a respeito do indicador VTI, o qual, por ser uma variável residual obtida a partir da diferença entre o Valor Bruto da Transformação Industrial (VBPI) e os custos das operações industriais (COI), sofre impacto da taxa de câmbio em função dos custos dos insumos importados. Assim, uma desvalorização (valorização) cambial leva a um aumento (redução) relativo dos COI sem, necessariamente, um aumento (redução) do VBPI e, consequentemente, uma redução (aumento) do VTI. No caso da indústria farmacêutica, em que a produção de medicamentos no Brasil é fortemente dependente de insumos importados, as variações cambiais tendem a impactar diretamente nos COI. Além disso, a variação dos preços dos insumos comprados no próprio país também pode influenciar os COI.

Juntamente com o impacto dos preços, a redução do VTI está ligada a característica do portfólio de medicamentos das empresas nacionais, que é fortemente concentrada em genéricos e similares apresentando um valor agregado inferior. As ETNs no Brasil, ao importarem cada vez mais medicamento acabados para comercialização no Brasil, reduziram suas atividades produtivas no país, sobretudo, aquelas de maior complexidade. Ademais, as ETNs localizadas no Brasil não empreendem atividades inovativas no país, uma vez que concentram o desenvolvimento de novos produtos nas suas sedes ou em seus centros de pesquisa em outros países. Tais empresas destinam apenas a parte da pesquisa de teste clínico de fase III $^{6}$

\footnotetext{
${ }^{6}$ As atividades de P\&D para desenvolvimento de um novo medicamento consistem em cinco etapas: descoberta de um novo composto, desenvolvimento pré-clínico, testes clínicos (fase I; fase II; fase III), registro e testes clínicos pós-mercado (fase IV) (BASTOS, 2005, apud PARANHOS, 2010).
} 
no Brasil. Assim, o setor farmacêutico brasileiro constituiu uma trajetória de grande dependência de tecnologia estrangeira, com baixos esforços inovativos e fraca interação entre as empresas e as instituições científicas. Já as empresas nacionais especializadas na produção de genéricos, apesar de terem depreendido esforços diferenciados para a atuação neste segmento, não conseguiram acumular capacidades inovativas o suficiente para desenvolver produtos novos (PARANHOS, 2010).

No que diz respeito aos esforços inovativos dessa indústria, os dados da Pesquisa de Inovação do IBGE (2016) mostram que a taxa de empresas farmacêuticas inovadoras é maior que a da indústria de transformação, $36 \%$ contra $52 \%$, em 2014. Contudo, a taxa de inovação das empresas farmacêuticas tem uma tendência declinante ao se comparar com 2008 (64\%) e 2011 (54\%). As empresas farmacêuticas brasileiras gastaram, em média, 2,6\% ( $\mathrm{R} \$ 1,4$ bilhão) da sua receita líquida de vendas em atividades de $\mathrm{P} \& \mathrm{D}$, considerando as atividades de P\&D internas $(2,2 \%$ ou $\mathrm{R} \$ 1,2$ bilhões) e externas $(0,4 \%$ ou $\mathrm{R} \$ 213$ milhões), em 2014. Esse valor fica muito aquém da média mundial do setor farmacêutico, que destina, aproximadamente, 20\% das receitas para P\&D (IEDI, 2010).

\section{A INSERÇÃO EXTERNA DA INDÚSTRIA FARMACÊUTICA BRASILEIRA}

A indústria farmacêutica brasileira se desenvolveu voltada ao mercado doméstico. Ao longo de sua formação, a indústria brasileira foi protegida por barreiras tarifárias e não-tarifárias, o qual garantiam reserva de mercado aos produtos nacionais. A partir do início da década de 1990, com a abertura comercial e diversas mudanças no arcabouço regulatório e institucional, as empresas farmacêuticas passaram por processos de reestruturação e de aumento da competição interna, devido à entrada de concorrentes estrangeiros, permanecendo os esforços direcionados ao mercado brasileiro.

A inserção internacional das empresas farmacêuticas teve impulso após os anos 2000, período em que ocorre o crescimento das empresas farmacêuticas nacionais, sobretudo aqueles fabricantes de medicamento genéricos, e os instrumentos de política em apoio à internacionalização foram aprimorados. O fortalecimento da competitividade internacional das empresas de capital nacional foi uma meta transversal presente nas três políticas industriais recentes - PITCE, PDP, PBM. O BNDES tem sido o principal instrumento do governo de apoio à internacionalização de empresas, ao lado da Agência Brasileira de Promoção de Exportações e Investimentos (Apex-Brasil). Nesse sentido, o BNDES atua em duas frentes: por meio do subprograma de exportação contido no Programa de Apoio ao Desenvolvimento do Complexo Industrial da Saúde (Profarma) e através da sua linha de internacionalização que financia os investimentos externos (BNDES, 2017).

Por meio da sua linha de internacionalização, o BNDES desembolsou R \$ 10,8 bilhões no período de 2005 a 2013 para empresas investirem em projetos de internacionalização ${ }^{7}$, sendo que menos de $1 \%$ deste total foi utilizado por uma empresa farmacêutica, segundo o Relatório de Operações de Internacionalização do BNDES (2014). Além dos recursos, o BNDES também oferece suporte aos investimentos de empresas no exterior por meio da sua Área Internacional, que conta com um escritório em Montevidéu, no Uruguai, e uma subsidiária em Londres, na Inglaterra. Outros dois instrumentos também proporcionam auxílio à internacionalização, porém com menor impacto aos investimentos externos. O BNDES-Exim, criado em 1990, financia os produtos exportados por meio de linhas de crédito à produção (pré-embarque) e à comercialização (pós-embarque). Este programa é orientado às empresas instaladas no Brasil que procuram destinar sua produção ao mercado externo e os produtos financiáveis são, preferencialmente, os que possuem maior valor agregado e que apresentam índice de nacionalização mínimo de $60 \%$. O Banco financia processos de capitalização, desenvolvimento, consolidação e internacionalização de empresas brasileiras e o reforço de suas estruturas de capital por meio de participações societária minoritárias, pelo programa BNDESPar, estabelecido em 2009 (PERIN, 2014).

No que se refere à Apex-Brasil, sua atuação está direcionada à promoção das exportações, através da promoção comercial, informações sobre os mercados externos e capacitação de empresas, e na atração de

\footnotetext{
${ }^{7}$ Os projetos de internacionalização apoiados pelo BNDES são aquisições, greenfield, expansão e abertura de filial.
} 
IDE. Em parceria com a Associação Brasileira da Indústria Farmoquímica e de Insumos Farmacêuticos (Abiquifi), a Apex-Brasil passou a coordenar, em 2009, um projeto para ampliar a inserção internacional da cadeia farmacêutica. Dentro do escopo dos projetos destacam-se o "projeto comprador" e o "projeto imagem sanitária". O primeiro é designado a realizar rodadas de negócios entre empresas estrangeiras e brasileiras com o objetivo de atrair potenciais compradores de produtos nacionais, enquanto o segundo projeto envolve a recepção de delegações estrangeiras, agências reguladoras, empresas farmacêuticas e instituições compradoras, para reuniões com a Anvisa, visitas às unidades fabris de empresas brasileiras e workshops setoriais (PIMENTEL et al., 2014).

\subsection{O desempenho do comércio exterior da indústria farmacêutica brasileira}

O desempenho do comércio exterior da indústria farmacêutica brasileira está relacionado com a dinâmica global do setor, bem como, com as mudanças estruturais do mercado doméstico. Em relação ao contexto global, IEDI (2016) aponta que há um fenômeno de commoditização da produção de IFAs, especialmente de insumos sem proteção de patentes, que são considerados não exclusivos. Tal fenômeno foi fortalecido pelas estratégias das empresas farmacêuticas de países desenvolvidos e se consolidou por meio de políticas públicas de incentivo à indústria farmacêutica nos países asiáticos.

Os principais países produtores de IFAs não exclusivos são a China e a Índia. As grandes empresas farmacêuticas têm seguido processos de internacionalização de parte da sua produção, principalmente a fabricação de farmoquímicos, para países que têm menores custos. Este processo foi motivado pelos custos cada vez maiores na descoberta de moléculas e pela reorientação estratégica das grandes empresas, que passaram a focar no desenvolvimento de novos medicamentos de base biotecnológica e nas atividades de marketing e comercialização. Assim, a organização produtiva da indústria farmacêutica sofreu uma mudança distributiva, em que os países em desenvolvimento passaram a produzir os IFAs não exclusivos (RADAELLI, 2006; MITIDIERI et al., 2015).

Embora a maioria da produção de IFAs ainda esteja concentrada em países desenvolvidos, uma vez que estão ligados à sua demanda global, os países em desenvolvimento evidenciam maior dinamismo na produção. Desta forma, a abundante oferta de IFAs com baixos preços no mercado internacional motivou o aumento da importação pelas fabricantes brasileiras de genéricos.

Além disso, grande parte das importações de medicamentos prontos é de base biotecnológica. Segundo IEDI (2016), 28,5\% do total do déficit comercial do setor farmacêutico são de medicamentos biotecnológicos. Isto porque, oito entre os 10 principais medicamentos acabados com maior valor de importação são obtidos por rota biotecnológica ${ }^{8}$. Cabe ressaltar que, neste caso, as capacidades para 0 desenvolvimento e produção interna são diferentes daquelas construídas pelas empresas farmacêuticas nacionais na última década, pois está mais próximo de um déficit de conhecimento (REIS; LANDIM; PIERONI, 2011). Segundo Torres (2015), a incorporação da produção de medicamentos biológicos pela indústria brasileira é prejudicada pela pouca experiência na fabricação e inovação de farmoquímicos, pois a natureza do processo produtivo de biofármacos envolve células vivas, que dificilmente é dissociado da formulação final do medicamento e requer competências de maior complexidade tecnológica.

\footnotetext{
${ }^{8}$ Devido à grande dependência externa do país em produtos biotecnológicos, o governo adotou uma política de promoção da produção doméstica desse segmento, a partir de 2008. O estabelecimento de Parceiras para o Desenvolvimento Produtivo para a transferência de tecnologia entre os laboratórios estrangeiros e os públicos e privados nacionais despertou o interesse das empresas nacionais aos medicamentos biológicos, mais especificamente, os biossimilares. Ações nesse sentido foram observadas com a criação de joint ventures, como a Bionovis, formada pelos laboratórios Aché, EMS, Hypermarcas e União Química, e a Orygen, composta pelos laboratórios Biolab, Cristália, Eurofarma e Libbs (GOMES, 2014). Posteriormente, os laboratórios Cristália e Libbs se separaram da Orygen para seguir individualmente. Esta política também procurou fortalecer os laboratórios públicos oficiais na produção de medicamentos de síntese química e biológicos e a integração com o segmento de insumos nacional, fármaco e biofármaco.
} 
Outro importante fator de pressão sobre a balança comercial do setor farmacêutico foi o crescimento do consumo doméstico de medicamentos pelo SUS e pela população em geral devido à ampliação da renda e à expansão das compras externas de medicamentos de marca (IEDI, 2016). A apreciação da taxa de câmbio deixou os medicamentos importados mais baratos para a população e para o SUS.

No que tange à oferta interna, embora a indústria farmacêutica brasileira tenha observado uma trajetória de forte ampliação, em que as empresas farmacêuticas de capital nacional conseguiram aproveitar as oportunidades e ampliaram significativamente sua participação no mercado brasileiro, o mesmo não se refletiu no saldo do comércio exterior, porque, na sua maioria, as empresas ficaram muito especializadas no mercado interno. Por outro lado, devido ao aumento da concorrência no mercado brasileiro com a ascensão das empresas nacionais de genéricos, as ETNs adotaram uma estratégia defensiva com o fechamento ou redução da fabricação local e importação dos medicamentos, estratégia que foi fortalecida pela apreciação do Real e ampliação da produção de medicamento biológico, que tende a ser concentrada (IEDI, 2016). Além disso, as ETNs adquiriram fabricantes nacionais de genéricos para garantir sua expansão no mercado brasileiro neste segmento.

Como pode ser visto na Figura 1Figura 1, a balança comercial da indústria farmacêutica é estruturalmente deficitária. Em 2016, o déficit de produtos farmoquímicos foi de US\$ 1,6 bilhão e de produtos farmacêuticos foi de US\$ 5,1 bilhões. As importações de produtos farmoquímicos e farmacêuticos se acentuaram com a abertura comercial iniciada em 1990, impulsionados pelas reduções das barreiras tarifárias e não-tarifárias. Até então, o mercado era protegido por barreiras que restringiam a entrada de produtos importados. No período entre 1990 e 2016, as compras externas de produtos farmoquímicos e farmacêuticos cresceram, em média, $8,3 \%$ e $15,1 \%$ ao ano, respectivamente. A partir de 2004, há uma intensificação da entrada de produtos importados que segue até 2014, sendo que o ápice das importações foi obtido em 2013, quando somaram US\$ 9,1 bilhões. Em termos de composição, a maior contribuição para o saldo negativo da indústria farmacêutica deve-se às compras de medicamento acabados.

Figura 1 - Evolução do comércio exterior de produtos farmoquímicos e medicamentos, 1991-2016* (em US\$ milhões)

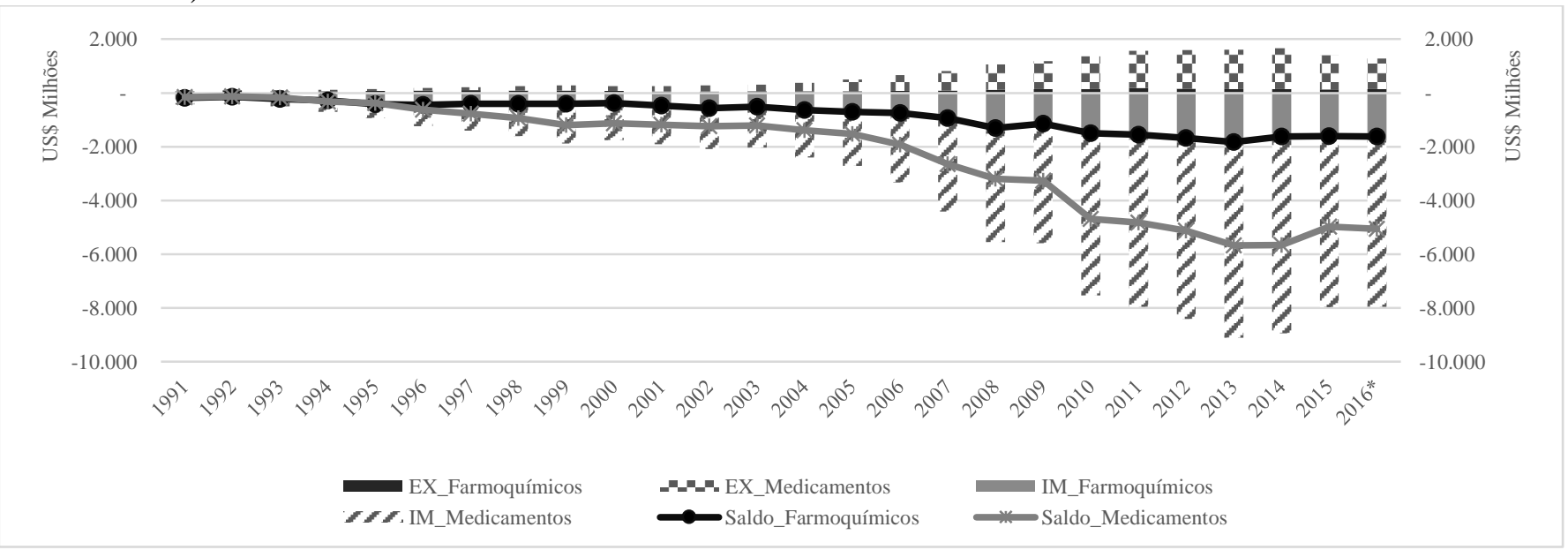

Fonte: MDIC/SECEX (2017).

Nota: * janeiro a outubro de 2016.

A Figura 2 apresenta a distribuição das importações brasileiras de produtos farmoquímicos e farmacêuticos por país de origem. Nota-se o expressivo crescimento dos produtos farmoquímicos importados da China, que representavam 7\% no intervalo de 1991-1995 e passaram para 27\% em 2011-2016. Os produtos farmoquímicos indianos também aumentaram sua participação de $1 \%$ para 6\%, na mesma base de comparação. Já os medicamentos acabados são, majoritariamente, comprados de países desenvolvidos. A Alemanha, juntamente com os Estados Unidos, são os principais países de origem, somando $36 \%$ do total dos medicamentos importados pelos Brasil. 
Figura 2 - Importações brasileiras de produtos farmoquímicos e medicamentos por país de origem, 1991$2016^{*}$
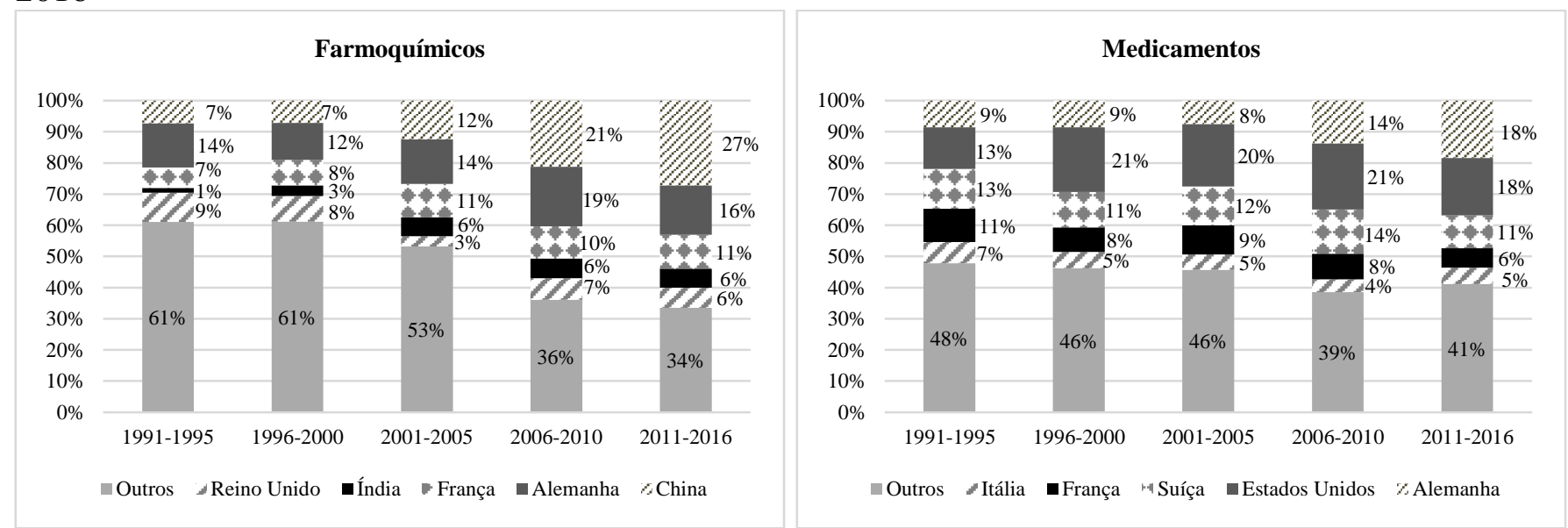

Fonte: MDIC/SECEX (2017).

Nota: * janeiro a outubro de 2016.

Do lado das exportações, nota-se um crescimento mais expressivo a partir de 2003, o que coincide com o período de expansão das empresas de capital nacional. A taxa de crescimento médio das exportações de produtos farmacêuticos foi semelhante à taxa de crescimento das importações no período entre 2003 a 2016 , $10,5 \%$ ao ano e 10,1\% ao ano, respectivamente. Porém, as vendas brasileiras externas de produtos farmoquímicos e farmacêuticos são de menor complexidade e têm menor peso na pauta exportadora. Em 2016, as exportações do setor farmacêutico representaram 0,8\% das exportações totais do Brasil, enquanto que as importações corresponderam a 6,9\%.

De acordo com Pimentel et al. (2014), a farmacêutica foi a atividade da indústria de transformação que teve maior crescimento da participação das exportações nas receitas das empresas brasileiras, $73 \%$ entre 2003 e 2013, chegando a representar $10 \%$ neste último ano. Até outubro de 2016, as vendas externas de farmoquímicos e medicamentos alcançaram US\$ 152 milhões e US\$1,1 bilhão, respectivamente. Porém, o ápice das exportações foi obtido em 2014, em que somaram US\$ 1,7 bilhão em conjunto, segundo os dados da Secex do MDIC (Figura 1). O aumento da presença das empresas farmacêuticas brasileiras em mercados estrangeiros pode ser considerado um dos principais motivos para o crescimento das exportações.

A Figura 3 mostra que os destinos das exportações de produtos farmoquímicos tornaram-se menos concentrados nos últimos anos, com o aumento da participação, principalmente, da China, Espanha e Canadá como receptores dos produtos brasileiros. Nos últimos dois períodos, 2006-2010 (20\%) e 20112016 (25\%), a participação do Canadá como destino das exportações de farmoquímicos cresceu consideravelmente. A empresa de capital nacional Blanver, terceira maior produtora de insumos farmacêuticos do mundo com participação de $13 \%$ no mercado global, obtém parte significativa de sua receita com exportação (FACTO, 2016). 
Figura 3 - Exportações brasileiras de produtos farmoquímicos e medicamentos por país de destino, 1991 $2016^{*}$
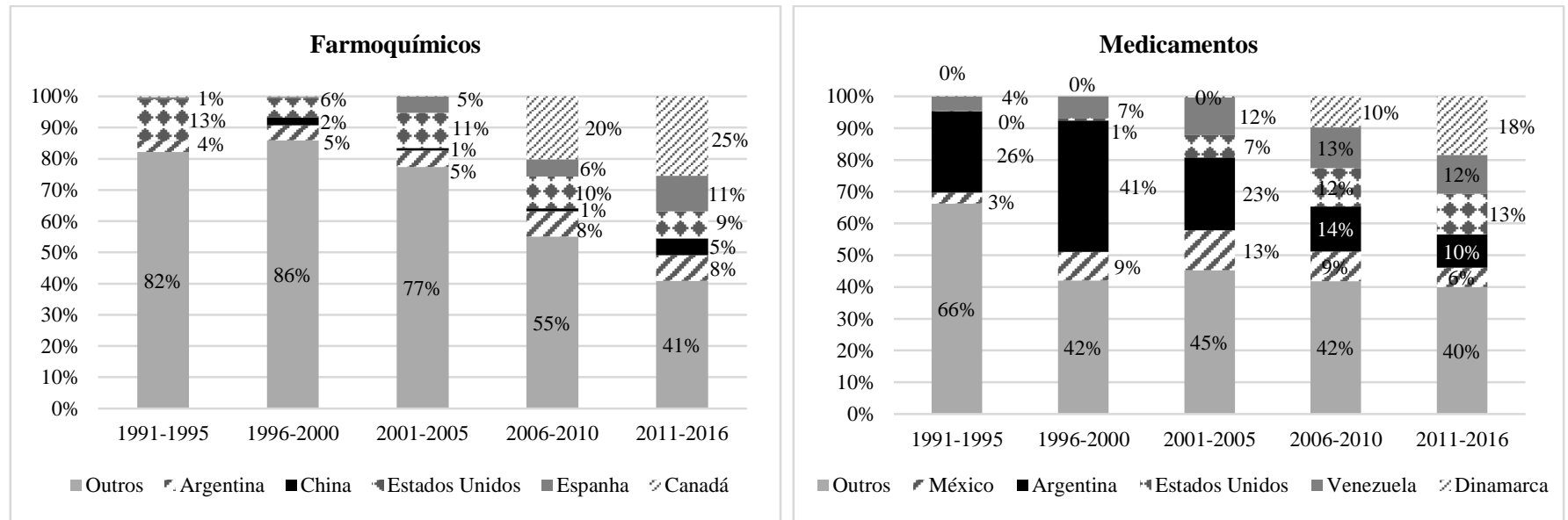

Fonte: MDIC/SECEX (2017).

Nota: * janeiro a outubro de 2016.

No caso dos medicamentos, destaca-se a maior participação da Dinamarca como destino das exportações, o que se deve à venda de insulina, cujo Brasil é uma provável plataforma de exportação da Novo Nordisk (PIMENTEL et al., 2014). O comércio intrafirma tem grande peso na produção farmacêutica mundial. As grandes empresas farmacêuticas estão presentes em praticamente todas as regiões do mundo e, na maioria das vezes, elas internacionalizam as etapas de maior valor agregado aos países desenvolvidos e, aos países em desenvolvimento, direcionam as etapas de fabricação ou distribuição de medicamentos (HASENCLEVER et al., 2010).

A diminuição relativa da Argentina como destino das exportações de medicamentos pode estar relacionada à compra de laboratórios argentinos por empresas brasileiras, as quais podem ter transferido parte da produção para atender à demanda daquele país. Apesar de a América Latina ter pouca participação entre os cinco principais destinos, os países vizinhos em conjunto compreendem mais da metade das vendas externas da indústria farmacêutica.

\subsection{As estratégias de internacionalização das empresas farmacêuticas brasileiras de capital nacional}

Nos últimos anos, um pequeno número de empresas farmacêuticas nacionais, mas com grande participação no mercado brasileiro, atingiu porte expressivo e capacidades que as tornaram capazes de alcançar a posição de empresas internacionalizadas. A Figura 4 exibe a evolução dos investimento brasileiros no exterior direcionados à indústria farmacêutica. Tanto o estoque de recursos, quanto o número de investidores cresceram significativamente entre 2009 e 2014, chegando a US\$ 84,3 milhões, porém caíram pela metade em 2015. 
Figura 4 - Estoque dos investimentos diretos e número de investidores da indústria farmacêutica brasileira no exterior, 2009-2015 (em US\$ milhões)

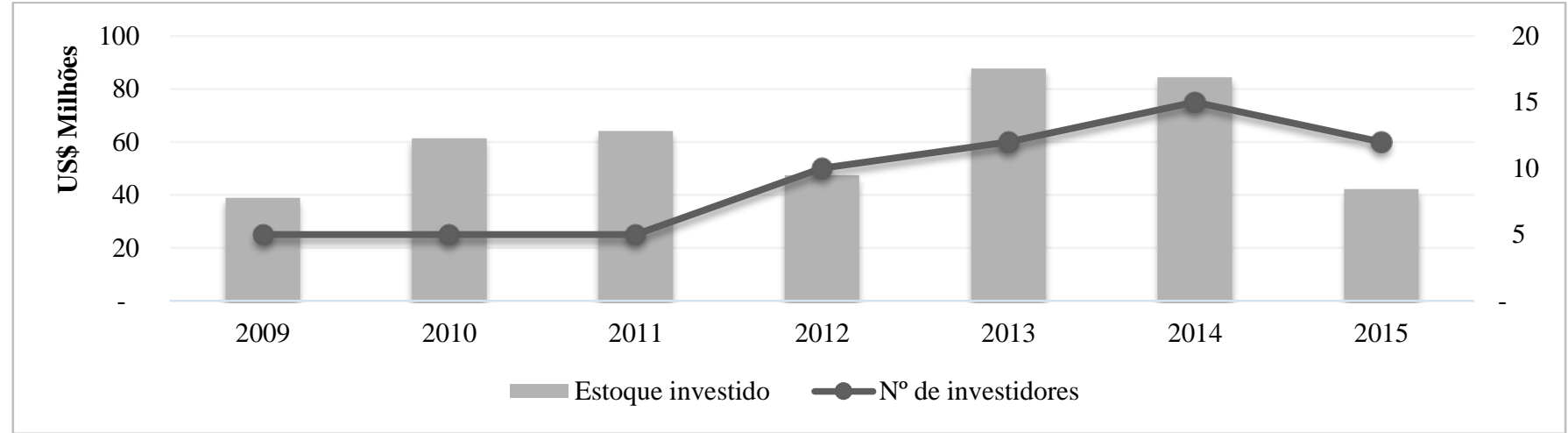

Fonte: Capitais Brasileiros no Exterior - Banco Central do Brasil (2016).

Conforme alguns estudos apontam (PIMENTEL et al.; 2014; HAAG; HENKIN, 2013; HAAG; STAROSTA, 2006), a exportação é identificada como o primeiro passo rumo à inserção externa das empresas farmacêuticas brasileiras. Os produtos exportados são dirigidos, principalmente, aos países latinoamericanos, assim como, os primeiros investimentos diretos das empresas. Diversos fatores influenciam na escolha dos países dessa região, os quais estão relacionados às vantagens competitivas de escala e qualidade sanitária em comparação com seus concorrentes latino-americanos. O tamanho do mercado brasileiro, que responde por $39 \%$ do mercado da região, é responsável por conferir vantagens de escala e de escopo às empresas brasileiras (IMS HEALTH, 2015 apud HYPERMARCAS, 2016a).

O elevado padrão regulatório exigido pela Anvisa, referente às informações necessárias para registro e à qualidade e segurança exigida das operações industriais no Brasil, concedem vantagem competitiva aos produtos farmacêuticos brasileiros. O manual de Boas Práticas de Fabricação da Anvisa é semelhante ao guia da União Europeia, o que proporciona maior segurança e previsibilidade em comparação com os concorrentes asiáticos. Devido a sua participação em diversos fóruns internacionais, a Anvisa é reconhecida como referência, principalmente, na América Latina, o que faz com que os países da região aceitem o dossiê brasileiro sem muitas alterações (PIMENTEL et al., 2014). Assim, os produtos brasileiros não precisam de muitos investimentos em adaptações ao entrar nos mercados vizinhos. Por outro lado, segundo a pesquisa realizada por Haag e Henkin (2013), as farmacêuticas nacionais também apontam que o longo tempo para registro de medicamentos na Anvisa faz com que o produto chegue ao país de destino muito tempo depois, o que implica em perda de competitividade.

A Eurofarma, por exemplo, tem a expectativa de cobrir $70 \%$ do mercado latino-americano e, para isso, mantém operações em diversos países da região (EUROFARMA, 2016). A Cristália exporta medicamentos acabados (anestésicos e antirretrovirais) e insumos farmacêuticos para mais de 30 países, entre América Latina, Ásia, África e Oriente Médio (CRISTÁLIA, 2016).

O Quadro 1 apresenta as estratégias de internacionalização das empresas farmacêuticas nacionais com inserção externa. 
Quadro 1 - Estratégias das empresas farmacêuticas brasileiras internaiconalizadas

\begin{tabular}{|c|c|c|c|}
\hline Empresa & Estratégia & Forma de inserção & Localização \\
\hline Eurofarma & Acessar mercado & Exportação/Aquisição & $\begin{array}{l}\text { Argentina, Uruguai, Bolívia, } \\
\text { Chile, Venezuela, Colômbia e } \\
\text { Guatemala }\end{array}$ \\
\hline Cristália & Acessar mercado & Exportação/Aquisição & $\begin{array}{l}\text { América Latina, Ásia, África e } \\
\text { Oriente Médio }\end{array}$ \\
\hline EMS & Acessar mercado & Exportação/Licenciamento & Polônia \\
\hline Hebron & Acessar mercado & IDE (greenfield) & EUA \\
\hline Libbs & Acessar mercado & Exportação & União Europeia \\
\hline Aché & Acesso a recursos & Acordos colaborativos & México \\
\hline EMS & Acessar ativos estratégicos & Acordos colaborativos & Itália \\
\hline Recepta & Acessar ativos estratégicos & Acordos colaborativos & EUA \\
\hline Biolab & Acessar ativos estratégicos & IDE & EUA \\
\hline Hebron & Acessar ativos estratégicos & Acordos colaborativos & $\begin{array}{l}\text { EUA, Portugal, Áustria e } \\
\text { Inglaterra }\end{array}$ \\
\hline União Química & Acessar ativos estratégicos & Acordos colaborativos & França \\
\hline EMS & Acessar tecnologia & IDE (greenfield) & EUA \\
\hline Biolab & Acessar tecnologia & IDE (greenfield) & Canadá \\
\hline Orygen e Bionovis & Aumentar a P\&D e tecnologia & $\begin{array}{l}\begin{array}{l}\text { Licenciamento } \\
\text { (greenfield) }\end{array} \\
\end{array}$ & Não disponível \\
\hline Aché & Aumentar a P\&D e tecnologia & IDE (greenfield) & Espanha \\
\hline
\end{tabular}

Fonte: Elaboração própria com base em EMS (2016); Eurofarma (2016); Biolab (2016); Cristália (2016); Recepta (2016); União Química (2016); Aché (2016); Hebron (2016); Libbs (2016); Haag e Henkin (2013).

Além das exportações para a América Latina, também há casos de farmacêuticas nacionais que conseguiram criar vantagens competitivas para exportar para países desenvolvidos. O laboratório Libbs que, em 2010, obteve a certificação de Conformidade da Farmacopeia Europeia passou a exportar o gestodeno para os países da União Europeia (LIBBS, 2016). A EMS tem acordos de licença e distribuição na Polônia, desde 2011 (EUROFARMA, 2016). O laboratório Hebron vende para o mercado norteamericano desde 2009 e, em 2011, abriu uma subsidiária nos EUA para facilitar a comercialização de medicamentos (Hebron, 2016).

Haag e Henkin (2013) identificaram os principais fatores motivadores na realização das exportações das empresas farmacêuticas de capital nacional ou predominantemente nacional. Entre eles destaca-se a ideia de que as empresas exportadoras são consideradas mais atrativas para contratos de licenciamento com empresas estrangeiras e constroem uma imagem de competitividade e eficiência no mercado global junto aos parceiros internacionais de pesquisa e transferência de tecnologia. Ademais, devido às diferentes normas regulatórias e estrutura de mercado de outros países, a exportação também é vista como uma forma de aprendizado e alinhamento da empresa nesses pontos.

A construção de vantagens competitivas no mercado doméstico parece ter motivado as empresas de capital nacional a seguirem estratégias mais dinâmicas de internacionalização. Em busca de ampliação de mercado e de melhor aproveitamento da capacidade instalada no Brasil, as farmacêuticas nacionais têm realizado investimentos em outros países.

Com a estratégia de internacionalização de acessar os mercados dos países vizinhos, a Eurofarma que adquiriu os laboratórios na Argentina, Uruguai, Bolívia, Chile, Venezuela, Colômbia e Guatemala entre 2009 e 2014 e a Cristália que adquiriu um laboratório argentino, em 2012. A proximidade cultural e geográfica juntamente com as vantagens competitivas mencionas anteriormente são os fatores que, possivelmente, influenciaram na decisão das empresas farmacêuticas nacionais a seguir tal estratégia. 
Há casos também de empresas farmacêuticas nacionais que estão se inserindo em países desenvolvidos. Tais empresas estão longe de conseguirem competir em condições de equidade com as grandes empresas farmacêuticas transnacionais, porém estão conseguindo entrar em mercados mais desenvolvidos por meio de aquisições compatíveis com seu porte. A atuação nesses mercados nem sempre segue a estratégia de expansão da empresa, mas é vista como uma possibilidade de atuar onde a oferta científica e tecnológica de fato ocorre (PALMEIRA FILHO; CAPANEMA, 2010).

Nesse sentido, são identificadas empresas farmacêuticas que se inserem em países desenvolvidos com estratégias de aprimoramento de ativos. Ao invés de instalar atividades de produção, tais empresas estão em busca de parcerias de P\&D para transferir o conhecimento adquirido na internacionalização ao sistema de inovação brasileiro (PIMENTEL et al., 2014). Haag e Henkin (2013) ressaltam que estes projetos têm servido para que as farmacêuticas nacionais se insiram na comunidade de pesquisa internacional. São vistos movimentos como estes nas empresas EMS, a qual firmou, em 2006, parceria com um laboratório italiano para intercambio de conhecimento e tecnologia, e a Achè, que estabeleceu uma aliança estratégica, em 2010, com uma empresa mexicana para pesquisa clínica de novos produtos e associações medicamentosas (EMS, 2016; ACHË; 2016). Outros casos de destaque se referem à startup Recepta, que foi criado através de parcerias de codesenvolvimento no exterior com o Ludwig Institute for Cancer Research do Massachusetts Institute of Technology (MIT), a Biolab, que instalou uma unidade de negócios nos EUA para captar parcerias com ETNs, e a Hebron, que mantém parcerias com EUA, Portugal, Áustria e Inglaterra (SILVA, 2011; BIOLAB, 2016; HEBRON, 2016).

Haag e Henkin (2013) também ressaltam os casos dos grupos Orygen e Bionovis, que têm intensificado seus projetos de inovação em parceria com centros de pesquisa estrangeiros para conduzir o lançamento de produtos brasileiros novos no mercado internacional. Além de planejar a comercialização desses medicamentos, por meio de plataformas próprias de distribuição regional ou de ETNs que tenham capacidade de atender globalmente.

Também são observados casos de empresas que estão conseguindo aproveitar as suas vantagens competitivas e buscando acessar conhecimentos nos sistemas de inovação dos países desenvolvidos. Segundo Haag e Henkin (2013), as farmacêuticas nacionais também se preocupam em estar presente no portfólio das empresas de produtos inovadores que poderão ser lançados nos próximos anos e terão impacto na participação das vendas externas sob o faturamento total da empresa. Em 2013, a EMS instalou uma subsidiária nos EUA, a Brace Pharma, com o objetivo de investir em medicamentos inovadores. A Biolab instalou um centro de P\&D no Canadá, em busca de incentivos mais consistentes para a inovação. A União Química realiza operações com o laboratório francês Thea desde 2009 e a Achè criou um centro de P\&D na Espanha, em 2010.

\section{CONCLUSÃO}

O presente trabalho buscou levantar as características do comércio exterior do setor farmacêutico e as estratégias de internacionalização em curso das empresas farmacêuticas nacionais, com base em dados secundários. Encontrou-se que o déficit comercial do setor é significativo e crescente e que a inserção comercial internacional das empresas farmacêuticas nacionais ainda é muito pequena. No entanto, identificou-se que algumas dessas empresas, que cresceram com a produção de genéricos para venda no mercado interno, estão expandindo sua atuação e iniciando estratégias de inserção internacional por meio de acesso a mercados e busca por capacitação tecnológica.

No geral, as empresas farmacêuticas nacionais que adotam uma estratégia de internacionalização para acessar mercados estão se inserindo, principalmente, nos países latino-americanos. As empresas farmacêuticas nacionais foram capazes de acumular capacidades que lhes conferem vantagens competitivas de escala e qualidade sanitária frente às concorrentes dos países vizinhos, o que facilita a inserção internacional em seus mercados. 
Por sua vez, as empresas farmacêuticas nacionais que buscam capacitação tecnológica no mercado internacional se inserem em países desenvolvidos. Estas empresas alcançaram porte expressivo, capacidade organizacional e possuem estratégias inovativas, que as permitiram se inserir em mercados mais desenvolvidos com estratégias de busca por tecnologias avançadas e diversificação do portfólio. Porém, ainda não é conhecido até que ponto tais empresas estão criando mecanismos de absorção do conhecimento obtido no exterior de modo a sustentar o processo de inovação.

Entende-se que ambas as estratégias de internacionalização são importantes para o fortalecimento da indústria farmacêutica brasileira. A atuação em diferentes mercados pode conferir maior poder de barganha às empresas e aumentar o potencial dos investimentos em P\&D. Além disso, a estrutura concorrencial na indústria farmacêutica é marcada pelo deslocamento contínuo da fronteira tecnológica, cujos desenvolvimentos estão dispersos globalmente. Deste modo, ao buscar a inserção externa, as empresas farmacêuticas têm maiores possibilidades de aumentar a sua capacidade tecnológica.

Visto que os resultados obtidos por este estudo suscitam diversas questões que os dados secundários não permitem responder, seja pela ausência de indicadores, seja pelo foco do estudo ser um fenômeno contemporâneo que ainda não é possível encontrar estudos a respeito, planeja-se a realização de uma pesquisa de campo ainda em 2017 para obter-se evidências primárias junto às empresas. Entre os objetivos que serão buscados estão: a) detectar se a especialização das empresas farmacêuticas nacionais na fabricação de medicamentos genéricos e medicamentos com baixa capacidade inovativa, os quais possuem baixo valor agregado e baixa competitividade internacional, é um obstáculo ou uma vantagem competitiva à internacionalização de empresas; b) buscar entender como as empresas estão absorvendo o conhecimento criado nos países desenvolvidos e se este tem impacto na sua base produtiva e tecnológica. Para tanto, demais elementos a respeito da característica da internacionalização das empresas farmacêuticas nacionais, além das estratégias, precisam ser estudados, tais como as formas de entrada, as vantagens competitivas e os obstáculos encontrados neste processo.

\section{REFERÊNCIAS}

ACHÉ. Disponível no: 〈http://www.ache.com.br/>. Acesso em 15 nov. 2016.

BANCO NACIONAL DE DESENVOLVIMENTO ECONÔMICO E SOCIAL - BNDES. Relatório de Operações de Internacionalização. Tabulação especial. Disponibilizado em mar. 2014.

BANCO NACIONAL DE DESENVOLVIMENTO ECONÔMICO E SOCIAL - BNDES. Disponível no: <http://www.bndes.gov.br>. Acesso em 20 jan. 2017.

BERMUDEZ, J. et al. O Acordo TRIPS da OMC e a Proteção Patentária no Brasil: mudanças recentes e implicações para a produção local e o acesso aos medicamentos. Rio de Janeiro: Fiocruz/ENSP, 2000.

BIOLAB. Disponível no: 〈http://www.biolabfarma.com.br/>. Acesso em 15 nov. 2016.

BRASIL. BANCO CENTRAL DO BRASIL - BCB. Capitais Brasileiros no Exterior 2007-2015. Acesso em 15 jan. 2017.

BRASIL. LEI No 9.787, DE 10 DE FEVEREIRO DE 1999. Regulamento Altera a Lei no 6.360, de 23 de setembro de 1976, que dispõe sobre a vigilância sanitária, estabelece o medicamento genérico, dispõe sobre a utilização de nomes genéricos em produtos farmacêuticos e das outras providências. Brasília, 10 fev. 1999. Disponível em: <http://www.planalto.gov.br/ccivil_03/leis/19787.htm>. Acesso em: 15 jan. 2016. 
BRASIL. MINISTÉRIO DO DESENVOLVIMENTO, INDÚSTRIA E COMÉRCIO EXTERIOR MDIC. Secretaria de Comércio Exterior, Sistema AliceWeb. Disponível no:

$<$ http://aliceweb.mdic.gov.br>. Acesso em jan. 2017.

BUCLEY, P.; CASSON, M. C. The future of multinational enterprise. Londres: Homes \& Meier, 1976.

CRISTÁLIA. Disponível no: < http://www.2cristalia.com.br/>. Acesso em 15 nov. 2016.

CUERVO-CAZURRA, A.; GENC, M. Transforming disadvantages into advantages: developing country MNEs in the least developed countries. Journal of International Business Studies , v. 39, p. 957-979, 2008.

DUNNING, J. The eclectic paradigm as an envelope for economic and business theories of MNE activity. International Business Review, Reino Unido, n. 1, v. 9, p. 163-190, 2000.

DUNNING, J. Trade, Location of Economic Activity and the Multinational Enterprise: a search for an eclectic approach. In: DUNNING, J. Explaining International Production. Londres: Unwin Hyman, cap. 1, p. 13-40, 1988.

DUNNING, J.; LUNDAN, S. Multinational Enterprises and the Global Economy. EUA, Reino Unido: Edward Elgar Publishing, 2008.

EMS. Disponível no: <http://www.ems.com.br/>. Acesso em 15 nov. 2016.

EUROFARMA. Disponível no: < http://www.eurofarma.com.br/>. Acesso em 15 nov. 2016.

FACTO. Blanver aposta na internacionalização de sua divisão de medicamentos. 5 jan. 2016. Disponível em: <http://abiquifi.org.br/clipping/blanver-aposta-na-internacionalizacao-de-sua-divisao-demedicamentos/>. Acesso em 01 fev. 2017.

FINOTTI, O. Medicamentos genéricos no Brasil: avanços e desafios. II Enifarmed $-2^{\circ}$ Encontro Nacional de Inovação em Fármacos e Medicamentos. São Paulo, 12-13 ago. 2008.

GOMES, E. B. P. Clusters e biotecnologia para a superação da imitação: estudo de caso da indústria farmacêutica brasileira. 2014. 376 f. Tese (Doutorado) - Programa de Pós-Graduação em Políticas Públicas, Estratégias e Desenvolvimento, Instituto de Economia, Universidade Federal do Rio de Janeiro, Rio de Janeiro, 2014.

HAAG, V.; HENKIN, H. Ampliando a inserção internacional dos setores farmoquímico e farmacêutico brasileiros: alternativas estratégicas. São Paulo: SINDUSFARMA, 2013.

HAAG, V.; STAROSTA, E. Oportunidades no Mercado Internacional para a Indústria Farmacêutica Brasileira: análise da dinâmica do setor e por produto. Estudos FEBRAFARMA, 2006.

HASENCLEVER et al. Desafios de operação e desenvolvimento do Complexo Industrial da Saúde. Rio de Janeiro: E-papers, 2016.

HASENCLEVER, L. (org.) Economia Industrial: Fundamentos teóricos e práticas no Brasil. 2 ed. Rio de Janeiro: Elsevier, 2013, p. 193-2010.

HEBRON. Disponível no: < http://www.hebron.com.br/> Acesso em 15 nov. 2016. 
HYPERMARCAS. Disponível no: < http://www.hypermarcas.com.br/>. Acesso em 15 nov. 2016.

HYPERMARCAS. Relações com Investidores. Formulário de Referência 2016 - Hypermarcas S.A, 2016a.

INSTITUTO BRASILEIRO DE GEOGRAFIA E ESTATÍSTICA - IBGE. Pesquisa Industrial de Inovação Tecnológica 2014. Rio de Janeiro: IBGE, 2016.

INSTITUTO BRASILEIRO DE GEOGRAFIA E ESTATÍSTICA - IBGE. Pesquisa Industrial Anual 2014. Rio de Janeiro: IBGE, 2017.

INSTITUTO DE ESTUDOS PARA O DESENVOLVIMENTO INDUSRTIAL - IEDI. Panorama mundial e brasileiro do setor farmacêutico, dez. 2016.

INSTITUTO DE ESTUDOS PARA O DESENVOLVIMENTO INDUSTRIAL - IEDI. Carta IEDI $n^{\circ}$ 411. Publicada em 19 de abr. de 2010.

KUEMMERLE, W. The drivers of foreign direct investment into research and development: an empirical investigation. Journal of International Business, v. 30, n. 1, p. 1-24, 1999.

LE BAS, C.; PATEL, P. The determinants of home-base-augmenting and home-base-exploiting Technological activities: some new results on multinationals' locational strategies. SPRU Electronic Working Paper Series, n. 164, 2007.

LIBBS. Disponível no: <https://www.libbs.com.br/>. Acesso em 15 nov. 2016.

McKELVEY, M.; ORSENIGO, L. Pharmaceuticals as a Sectoral Innovation System. Paper prepared for the ESSY Project (European Sectoral Systems of Innovation) and within the Epris Project, 2001.

MITIDIERI, T. et al. Há espaços competitivos para a indústria farmoquímica brasileira? Reflexões e propostas para políticas públicas. BNDES Setorial, Rio de Janeiro, n. 41, p. 43-78, mar. 2015

NARULA, R.; ZANFEI, A. Globalization of innovation: the role of multinational enterprises. In: FAGERBERG, J.; MOWERY, D.; NELSON, R. The Oxford Handbook of Innovation. 1. ed. [S.1.]: Oxford University Press, 2004. Cap. 12, p. 318-346.

PALMEIRA FILHO, P.; CAPANEMA, L. A indústria farmacêutica nacional: desafios rumo à inserção global. In: ALÉM, A. C.; GIAMBIAGE, F. O BNDES em um Brasil em transição, Rio de Janeiro: BNDES, 2010, cap. 19.

PARANHOS, J. Interação entre Empresas e Instituições de Ciência e Tecnologia no Sistema Farmacêutico de Inovação Brasileiro: estrutura, conteúdo e dinâmica. 2010. 327 f. Tese (Doutorado) Curso de Economia, Instituto de Economia, Universidade Federal do Rio de Janeiro, Rio de Janeiro, 2010.

PAVITT, K. Sectorial patterns of technical change: towards a taxonomy and a theory. Research Policy, v. 13, p. 343-373, 1984.

PERIN, F. S. Trajetórias de desenvolvimento distintas: estudo sobre o padrão do Investimento Direto no Exterior do Brasil e da Coreia do Sul. 313 f. Dissertação (Mestrado) - Programa de Pós-Graduação em Economia, Universidade Federal de Santa Catarina, Florianópolis (SC), 2014. 
PIMENTEL, V. P. et al. Inserção internacional das empresas farmacêuticas: motivações, experiências e propostas para o BNDES. BNDES Setorial, Rio de Janeiro, n. 40, p. 5-42, set. 2014.

PINHEIRO, A. C.; ALMEIDA, G. B. de. Padrões setoriais da proteção na economia brasileira. Rio de Janeiro: IPEA. Texto para discussão, n. 355, 1994.

RADAELLI, V. A inovação na indústria farmacêutica: forças centrípetas e forças centrífugas no processo de internacionalização. Campinas: IGE/UNICAMP, 2006. (Dissertação de Mestrado). RAMAMURTI, R. Why study emerging-market multinationals? In: RAMAMURTI, R.; SINGH, J. Emerging Multinationals in Emerging Markets. Cambridge : Cambridge University Press, 2009. Cap. 1, p. 3-22.

RECEPTA. Disponível no: < http://www.receptabiopharma.com.br/>. Acesso em 15 nov. 2016.

REIS, C.; LANDIM, A. B.; PIERONI, J. P. Lições da experiência internacional e propostas para incorporação da rota biotecnológica na indústria farmacêutica brasileira. BNDES Setorial, Rio de Janeiro, n. 34, p. 5-44, 2011.

RUGMAN, A. Inside the multinationals: The economics of internal markets. Nova York: Columbia Press, 1981.

SILVA, D. Internacionalização Born Global: perspectivas para um novo modelo de desenvolvimento das empresas nacionais. Anais... I Circuito de Debates Acadêmicos. IPEA/CODE, 2011.

TEECE, D. A dynamics capabilities-based entrepreneurial theory of the multinational enterprise. Journal of International Business Studies, v. 45, p. 8-37, 2014.

TEECE, D. Explicating Dynamics Capabilities: the nature and microfoundations of (sustainable) enterprise performance. Strategic Management Journal, v. 28, p. 1319-1350, 2007.

TORRES, R. L. Capacitação tecnológica na indústria farmacêutica brasileira. 2015. 212 f. Tese (Doutorado) - Curso de Programa de Pós-graduação em Economia, Instituto de Economia, Universidade Federal do Rio de Janeiro, Rio de Janeiro, 2015.

TORRES, R.; CAVALIERI, H. Uma crítica aos indicadores usuais de desindustrialização no Brasil. Revista de Economia Política, v. 35, n. 4, p. 859-877, out./dez. 2015.

UNIÃO QUÍMICA. Disponível no: < http://www.uniaoquimica.com.br/>. Acesso em 15 nov. 2016.

UNITED NATIONS CONFERENCE ON TRADE AND DEVELOPMENT - UNCTAD. Investor

Nationality: Policy Challenges. World Investment Report. Nova York, Geneva: United Nations, 2016.

URRACA-RUIZ, A. U.; PARANHOS, J. O desenvolvimento de competências tecnológicas no setor farmacêutico pós-TRIPS: diferenças entre Brasil, Índia e China. In: ENCONTRO NACIONAL DE ECONOMIA, 40., 2012, Porto de Galinhas (PE). Anais... . ANPEC, 2012. p. 1-16.

VERNON, R. International investment and international trade in the product cycle. Quarterly Journal of Economics, v. 80, p. 190-207, 1966. 